\title{
The Role of Autophagy in Mechanical Stress-Induced Cell and Tissue Response
}

\author{
Liang Song ${ }^{1}$, Tengyu Zhang ${ }^{1}$, Xinying Shan ${ }^{1}$ and Yanghui Xing ${ }^{2 *}$ \\ ${ }^{1}$ National Research Center for Rehabilitation Technical Aids, China \\ ${ }^{2}$ Department of Biomedical Engineering, Shantou University, China
}

*Corresponding author: Yanghui Xing, 243 University Road, Shantou University, Shantou, 515063, China

\section{ARTICLE INFO}

Received: December 12, 2020

Published: 幽 December 21, 2020

Citation: Liang Song, Tengyu Zhang, Xinying Shan, Yanghui Xing. The Role of Autophagy in Mechanical Stress-Induced Cell and Tissue Response. Biomed J Sci \& Tech Res 32(4)-2020. BJSTR. MS.ID.005291.

\begin{abstract}
Cells need to respond and adapt to the changes of their surrounding physical environment for survival. On the other hand, autophagy is a catabolic mechanism for cells to cope with stress. But the relationship between cell autophagy and surrounding physical environment is poorly understood. In the past decade, accumulating number of literatures started to focus on the role of autophagy in cell mechanobiology, especially in myocardial cells, chondrocytes and endothelial cells. The results demonstrated that mechanical factors may lead to autophagy through PI3K-AKT-mTOR, oxygen free radical, AKT-FoxO and other pathways. The autophagic response of cells is a protective mechanism for cells to cope with their surrounding physical environment.
\end{abstract}

Keywords: Stress; Pathogen; Autophagy; Lysosome; Blood Flow; Eukaryotic Cells; Mechanical Stimuli; Morphology

\section{Introduction}

Autophagy means self-eating, and it is a natural and regulated mechanism of cells that remove unwanted or dysfunctional components, especially under external stimuli, such as pathogen invasion, starvation and hypoxia [1,2]. It allows orderly degradation and recycling of cellular components and exists in all eukaryotic cells. Autophagy is a highly conserved process that catabolizes intracellular components to maintain energy homeostasis and to protect cells against stress $[3,4]$. When autophagy occurs, the cell will first produce an isolation membrane structure called phagophore, which has a double-layer membrane structure. The phagophore will wrap the pathogens and damaged organelles in the cell through extension. After that, the autophagosome double membrane structure is formed to close the vesicle, and then the lysosome and the autophagosome are fused together to form the autolysosome. Finally, the fusion process is completed, and a monolayer structure is formed to degrade unnecessary cellular components $[5,6]$. After being degraded, the final autophagy lysosome structure will transport the degraded product out of autolysosome through the transfer protein on the membrane surface and supply it to the cell for energy or material recovery and reuse.
Through this process, the cells can respond to the lack of nutrients and energy under starvation conditions, and to achieve the self-supply of substances and energy. At the same time, it also can degrade some toxic substances to prevent their damaging effects to normal cells $[7,8]$. The process of autophagy is complicated, and there are still many details that we have not grasped. Other the other hand, cells and tissues within the human body are always in a certain physical environment [9]. For example, skeletal muscle is subjected to stretch; joint cartilage is under pressure; blood vessel endothelium cells are facing blood flow shear stress. Cells are able to respond to their surrounding mechanical environments, in which mechanical stimuli modulate cell proliferation, differentiation, morphology, migration and extracellular matrix production, as well as other physiological functions $[9,10]$. Cells need to adapt to their surrounding environments. Poor adaptation usually leads to pathological phenomena and occurrence of diseases. For example, myocardial hypertrophy is due to the heart muscle cells coping with long-term blood flow pressure or overloaded capacity. In serious cases, it may develop into heart failure [11]. 
Autophagy is an important mechanism for cells to cope with mechanical stimuli. Flow shear stress can promote endothelium cell autophagy activity and maintain its normal physiological activities. Abnormal stress can lead to poorly adapted pathological phenomena in tissues and cells. Autophagy is also closely related to fat, failed heart, degenerative lesions of joint cartilage and intervertebral discs $[12,13]$. Thus, in recent years, the influence of mechanical factors on cell autophagy has aroused great interests in researchers. This paper summarizes the effects of mechanical stimuli on the autophagy activity of different cells and tissues as well as relevant molecular mechanisms, and subsequently may provide theoretical basis for autophagy-related diseases (figure 1).

\section{A}

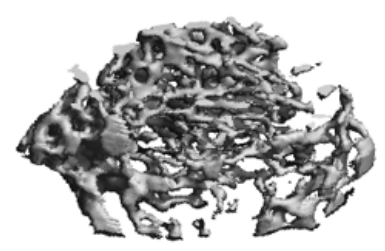

WT Mouse

B

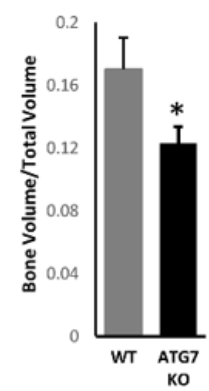

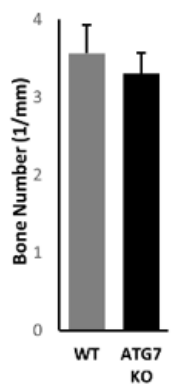

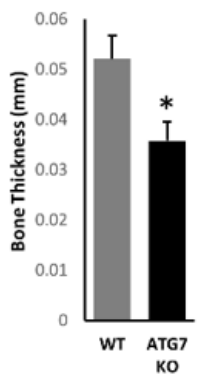

Figure 1: Bone structures of wild type and autophagy deficient mice (8 weeks of age). (A) micro-CT images; (B) Quantified parameters of trabecular bone of wild type and autophagy deficient mice. $\left(n=4,{ }^{*} p, 0.05\right)$. Error bars represent SEM.

\section{Literature Review}

\section{Effects of Mechanical Stimuli on Myocardial Cell Autophagy}

The heart is responsible for blood supply and it is always under strong blood flow pressures. If the myocardium is overloaded for a long time, left ventricular hypertrophy will occur. The symptoms include abnormal contraction, arrhythmia, abnormal energy metabolism and heart failure [14]. In this situation, the expression and activity of proteasome in heart muscles are significantly enhanced due to autophagy in ventricular hypertrophy [15]. Zhu, et al. [12] discovered for the first time the role of autophagy in pressure-overload-induced cardiac hypertrophy [12]. They used autophagy-reporter mice to confirm that pressure overload would cause increased autophagy activity continuously in cardiomyocytes. The increase in autophagy can be maintained for at least 3 weeks. Furthermore, other researchers have found that the autophagy activity of cardiomyocytes is resulted from stress due to blood pressure [16-18]. They also found that knocking out beclin1 can decrease the autophagy activity induced by blood pressure. On the other hand, overexpression of beclin 1 in mice leads to the opposite effects. Another study using atg5 knockout mice study confirmed the previous results [19]. Hariharan et al. [20] loaded primary rat cardiomyocytes cultured in vitro with a strain of $20 \%$ strain for $36 \mathrm{~h}$, and found that p62 degradation was significantly reduced, indicating an increase in autophagy activity [20]. Lin et al. [17] applied a strain of $20 \%$ to primary rat cardiomyocytes and COS7 cells in vitro for 48 hours. Subsequently, expression of LC3-II in cells increased, and the number of autophagosomes also increased significantly [17].

The effects of mechanical stimuli on cardiomyocyte autophagy are closely associated to proteasome activities. Pressure overload leads to an increase in the amount of misfolded protein in cardiomyocytes, and these proteins gradually gather around the nucleus to form aggregates, which are recognized and degraded by the autophagy system [16]. Pressure may cause accumulation of ubiquitinated proteins, aggregate formation and increased autophagy activity in left ventricular myocardial cells. Inhibiting cardiomyocyte proteasome activity in vitro makes ubiquitinated protein accumulate, and subsequently triggers autophagy. On the other hand, inhibiting autophagy activity of cardiomyocytes can effectively enhance the size and number of protein aggregates.

PI3K-AKT-mTOR pathway plays important role in regulating cell autophagy [21]. AKT regulates cardiomyocyte autophagy through its downstream transcription factor FoxO (forkhead box, class 0) protein family, which can promote autophagy activity [22]. When cardiomyocytes are under mechanical stimulation, the effects of on FoxOs are inhibited. Subsequently, activated Fox01 leads to cardiomyocyte ubiquitination and lysosome activities, and further 
enhances autophagy and protein degradation [23]. Additionally, Fox03 can activate downstream Bcl2 family member Bnip3, which inhibits the binding of $\mathrm{Bcl} 2$ and Beclin1 and promotes autophagy.

While pressure overload causes increased autophagy and hypertrophy of cardiomyocytes, there was also an increase of angiotensin II level in plasma and myocardium, and blood angiotensin II can promote autophagy activity in cells [24]. During pressure overload, expression of angiotensin II, phosphorylated PKCb1 and ERK1/2 are all increase significantly in cardiomyocytes [25]. Inhibiting anyone of them leads to inhibition of cardiomyocyte autophagy, suggesting the involvement of the AngII-PKCb1ERK1/2 pathway during this process. Angiotensin II can inhibit the expression of miR-34a in cardiomyocytes, which binds to Atg9A to inhibit autophagy activity. Angiotensin II type I receptor (AT1) mediates a variety of regulation of angiotensin II and plays an important role in myocardial hypertrophy induced by mechanical stress [26]. AT1 can mediate stress-induced autophagy independently of angiotensin II and p38 MAPK is involved in the process. However, ERK1/2 and JNK are not involved in the regulation of autophagy, in which AT1 plays an important role through PI3K-AKT signal pathway [17].

Cardiomyocyte autophagy is affected by mechanical stimuli and serves as a protective mechanism for cells to adapt their physical environments, subsequently to maintain their normal functions. The molecular mechanism of how mechanical stimuli induce cardiomyocyte autophagy is still unclear and needs further studies.

\section{Effects of Mechanical Stimuli on Chondrocyte Autophagy}

Articular cartilage is a soft connective tissue that covers the subchondral bone in a diathrodial joint. It is porous and filled with synovial fluid, which can flow in and out during motion and serves as a lubricating material to the joint. Mechanical stimuli have profound effects on the function of articular cartilage. Physiological level mechanical stress is beneficial for cartilage to maintain normal functions, while abnormal mechanical stress may result in chondrocyte death, extracellular matrix degradation and mineralization, osteoarthritis and other cartilage degenerative diseases [27]. During abnormal conditions, autophagy plays an important role in cartilage responses. Carames et al. [28] first studied the effect of mechanical shock on chondrocyte autophagy. They applied 40\% strain mechanical shock to cartilage tissues of cattle and human and found that the survival rate of cells was significantly reduced. They also found that sulfated glycosaminoglycans in extracellular matrix were gradually lost, and the expression of autophagy marker LC3-II in chondrocytes was increased in 24 hours. But the expression levels of ULK1, beclin1 and LC3-II were significantly reduced at $48 \mathrm{~h}$ and $96 \mathrm{~h}$. Pretreatment with autophagy inducer rapamycin can enhance autophagy activity, reduce cell death and the loss of sulfated glycosaminoglycans, indicating that autophagy may play a protective role in the early stage of chondrocytes subjected to mechanical shock $[28,29]$.
Ma et al. studied the effect of excessive mechanical pressure (1 MPa) on the autophagy of the colloidal nucleus pulposus cells (NP cells) in the center of the intervertebral disc and found that compared with the control group, the cell survival rate of the pressurized group was significantly reduced, and the ratio of cells that underwent autophagy increased significantly, and autophagy activity in cells also increased [13]. At the same time, oxygen free radicals accumulated in the cells during this process, suggesting that NP cells may activate autophagy through the oxygen free radical signaling pathway to cope with excessive mechanical stress. They also found that treating cells with autophagy inhibitor 3MA can significantly reduce the autophagy of NP cells. But at the same time, the occurrence rate of apoptosis increased significantly.

In another study, researchers investigated the effects of intermittent cyclic mechanical stretch on the calcification and autophagy of endplate chondrocytes. They found that chondrocyte autophagy activity was significantly increased on the 5th day, but significantly decreased on the 10th and 20th days [30]. Thus, short-term intermittent cyclic mechanical tension can promote chondrocyte autophagy, while long-term intermittent cyclic mechanical tension can inhibit autophagy. They also found that chondrocyte autophagy can protect cell from calcification. In chondrocytes, autophagy is a self-protection mechanism to respond to mechanical stimuli. When cartilage is under pressure, chondrocyte autophagy will increase in a short time. But if the stimulation lasts too long, the level of autophagy begins to decrease. Insufficient protection is gradually replaced by other mechanisms, such as apoptosis. Additionally, excessive mechanical stimulation may cause unregulated cell death.

\section{Effects of Mechanical Stimuli on Endothelial Cell Autophagy}

Endothelial cells are an important part of blood vessels, and they play an important role in maintaining the stability of blood vessel structure and functions. Under normal physiological conditions, endothelial cells are mainly affected by three mechanical factors: pressure, circumferential stretch and flow shear stress. Among them, flow shear stress affects gene expression, proliferation, migration, morphogenesis and adhesion of endothelial cells as well as their permeability and inflammation [31]. Autophagy is also affected by flow shear stress in order to maintain normal homeostasis in endothelial cells. It regulates vascular endothelial cell eNOS and ET-1 expression induced by laminar shear stress [32]. Additionally, different types and sizes of flow shear stress have different effects on endothelial cell autophagy. Laminar flow shear stress of $1.2 \mathrm{~Pa}$ or $2 \mathrm{~Pa}$ promoted endothelial cell autophagy activity, while the pathological type of oscillating flow does not promote autophagy. The magnitude of laminar shear stress also affects endothelial cell autophagy. Laminar shear stress of $0.4 \mathrm{~Pa}$ didn't promote endothelial cell autophagy [33]. Their results are consistent with previous study that the laminar shear stress of 0.5- 
1.5Pa promoted endothelial cell autophagy [32]. However, Ding et al. [34] showed that shear stress of $0.3 \mathrm{~Pa}$ can activate endothelial cell autophagy. When the magnitude of the flow shear stress increased to $3 \mathrm{~Pa}$, the effects on endothelial cell autophagy gradually disappeared [34].

Besides inducing endothelial cell autophagy, flow shear stress also enhanced expression of nitric oxide synthase and inhibited the expression of endothelin1 which play important roles in maintaining endothelial cell functions and vasoconstriction [35]. The use of autophagy inhibitors and inducers confirmed that autophagy modulates expression of nitric oxide synthase and endothelin1. In inflammatory states, the effects of flow shear stress on endothelial cell autophagy are enhanced [34]. When the autophagy pathway is blocked, flow shear stress can promote cytokine MCP-1 and interleukin-8 expressions, suggesting that endothelial cell autophagy promoted by flow shear force may have an anti-inflammatory effect. In addition, the flow shear stress promotes endothelial cell autophagy and is related to the intracellular oxidation-antioxidant balance [35].

The accumulation of oxygen free radicals was detected in endothelial cells stimulated by flow shear stress [33,35]. Sirt1, an intracellular sensor of redox activity, is induced by flow shear stress. It can sense the production and accumulation of oxygen free radicals which may induce autophagy. In addition, upregulation of Sirt1expression activates Fox01 and FoxO3, and subsequently induces the autophagy response. The expression of LOX-1 was also up-regulated, and LOX-1 could also activate autophagy through oxygen free radicals [34].

\section{Effects of Mechanical Stimuli on Autophagy of other Cells and Tissues}

Besides heart, cartilage and blood vessel, mechanical stress also can induce autophagy in skeletal muscle, and has great impact on skeletal muscle functions. Gumucio, et al. [36] found that autophagy related Vps34 and Beclin1 gene expression increased in rat skeletal muscle cells in rotator cuff tear rat model, indicating that autophagy was induced when skeletal muscle was under shear stress [36]. Ning et al. [37] obtained the similar results and they also found that proteoglycan inhibited the up-regulation of skeletal muscle autophagy [37]. In addition to macroautophagy, chaperonemediated autophagy is also involved in the influence of mechanical factors on skeletal muscle. Researchers found that after skeletal muscle cells were stimulated by stretch, chaperone-mediated autophagy was activated in order to maintain cell stability as a mechanism for transducing mechanical signals. Chaperonemediated autophagy was also responsible for activating a series of pathways and regulating skeletal muscle basic functions such as migration, adhesion, and proliferation. They also found that chaperone-mediated autophagy is an adaptive mechanism for skeletal muscle to resist acute exercise and repeated mechanical stimulation [38,39].
The autophagy of nerve cells is also affected by mechanical stress. The research in this area mainly revolves around the mechanical damage of nerve cells. When nerve cells are mechanically damaged (scratched), the level of autophagy is increased through the mTOR pathway. Additionally, compression injury can also increase the level of autophagy of nerve cells. In the early stage of cell damage, autophagy can inhibit apoptosis and protect nerve cells [40]. The mTOR pathway regulates the expression of Bax and $\mathrm{Bcl}-2$ by inhibiting apoptosis. In addition to macroautophagy pathways, chaperone mediated autophagy also participates in the process of nerve cells coping with mechanical damage [41]. In addition to the aforementioned cells and tissues, there are a small number of reports on the effects of mechanical stress on autophagy of fibroblasts, podocytes and bone cells. Due to the limitation of information, we will not review these reports in this article.

\section{Conclusion}

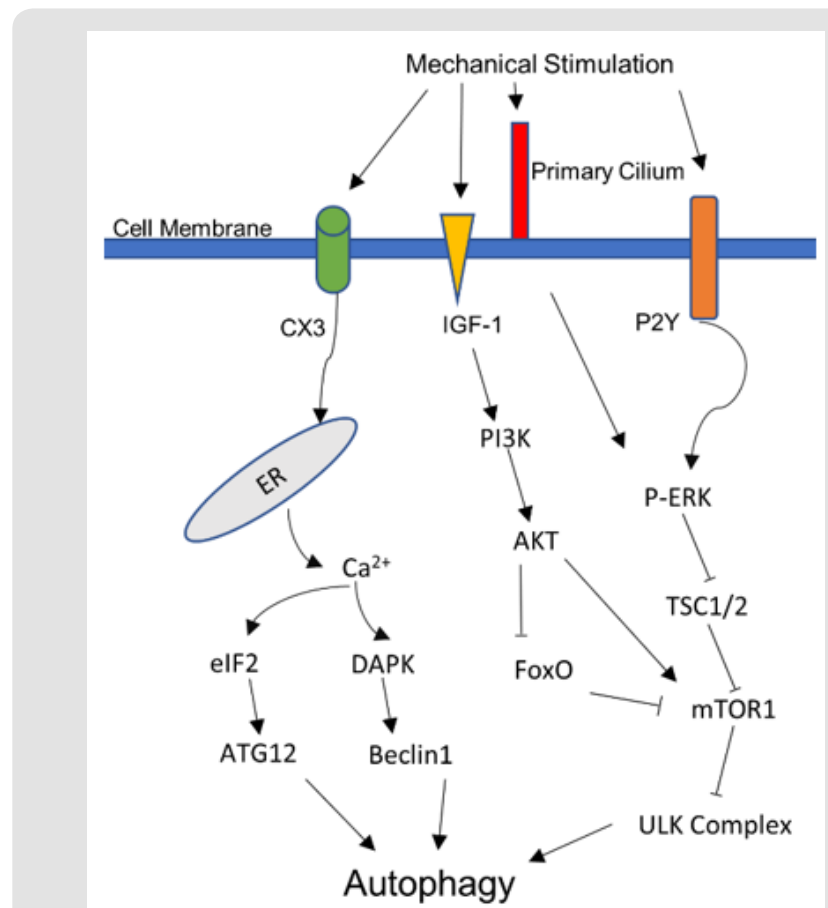

Figure 2: Illustration of various pathways connecting mechanical stimulation with cell autophagy.

In general, autophagy is a self-protective response of cells in response to mechanical stimuli. When subjected to mechanical stress at the physiological level, cells up-regulate the level of autophagy to maintain normal cell homeostasis, function and survival. Mechanical factors may cause autophagy through PI3KAKT-mTOR, oxygen free radical, AKT-FoxO and other pathways. The influence of mechanical factors on autophagy is closely related to many diseases, such as myocardial hypertrophy, atherosclerosis, cartilage degenerative diseases, and spinal cord injury. The current research has just started, and the influence of mechanical factors on autophagy remains to be clarified, and its regulatory mechanism is unclear. With further research to elucidate of the mechanism, it will 
help to understand the pathogenesis of these diseases and provide better prevention and treatment methods (figure 2).

\section{Conflicts of Interest}

The authors declare that they have no conflict of interests.

\section{Funding Statement}

This work was funded by Grant \#11672078 from the National Natural Science Foundation of China.

\section{References}

1. Ichimiya T, Yamakawa T, Hirano T, Yokoyama Y, Hayashi Y, et al. (2020) Autophagy and Autophagy-Related Diseases: A Review. Int J Mol Sci 21(23): 8974.

2. Dyshlovoy SA (2020) Blue-Print Autophagy in 2020: A Critical Review. Mar Drugs 18(9): 482.

3. Klionsky DJ, SD Emr (2000) Autophagy as a regulated pathway of cellular degradation. Science 290(5497): 1717-1721.

4. Fang Y, J Tan, Q Zhang (2015) Signaling pathways and mechanisms of hypoxia-induced autophagy in the animal cells. Cell Biol Int 39(8): 891898.

5. Reggiori F (2006) Membrane origin for autophagy. Curr Top Dev Biol 74: 1-30.

6. Klionsky DJ, Abdelmohsen K, Abe A, Abedin J, Abeliovich H, et al. (2016) Guidelines for the use and interpretation of assays for monitoring autophagy (3rd edition). Autophagy 12(1): 1-222.

7. D'Arcy MS (2019) Cell death: a review of the major forms of apoptosis, necrosis and autophagy. Cell Biol Int 43(6): 582-592.

8. Eskelinen EL (2019) Autophagy: Supporting cellular and organismal homeostasis by self-eating. Int J Biochem Cell Biol 111: 1-10.

9. Romani P, Lorea Valcarcel-Jimenez, Christian Frezza, Sirio Dupont (2020) Crosstalk between mechanotransduction and metabolism. Nat Rev Mol Cell Biol 20: 22-38.

10. Liu X, F Nakamura (2020) Mechanotransduction, nanotechnology, and nanomedicine. J Biomed Res p. 1-10.

11. Krueger W, Bender N, Haeusler M, Henneberg M (2020) The role of mechanotransduction in heart failure pathobiology-a concise review. Heart Fail Rev.

12. Zhu H, Tannous P, Janet L Johnstone, Kong Y, John M Shelton, et al. (2007) Cardiac autophagy is a maladaptive response to hemodynamic stress. Clin Invest 117(7): 1782-1793.

13. Ma KG, ZW Shao, SH Yang, J Wang, BC Wang, et al. (2013) Autophagy is activated in compression-induced cell degeneration and is mediated by reactive oxygen species in nucleus pulposus cells exposed to compression. Osteoarthritis Cartilage 21(12): 2030-2038.

14. Rothermel BA, JA Hill (2008) Autophagy in load-induced heart disease. Circ Res 103(12): 1363-1369.

15. Depre C, Wang Q Yan L, Hedhli N, Peter P, et al. (2006) Activation of the cardiac proteasome during pressure overload promotes ventricular hypertrophy. Circulation 114(17): 1821-1828.

16. Tannous P, Zhu H, Nemchenko A, Jeff M Berry, Janet L Johnstone, et al. (2008) Intracellular protein aggregation is a proximal trigger of cardiomyocyte autophagy. Circulation 117(24): 3070-3078.

17. Lin L, Liu X, Jianfeng Xu, Liqing Weng, Jun Ren, et al. (2015) Highdensity lipoprotein inhibits mechanical stress-induced cardiomyocyte autophagy and cardiac hypertrophy through angiotensin II type 1 receptor-mediated PI3K/Akt pathway. J Cell Mol Med 19(8): 1929-1938.
18. Fu L, Wei CC, Powell PC, Bradley WE, Collawn JF, et al. (2015) Volume overload induces autophagic degradation of procollagen in cardiac fibroblasts. J Mol Cell Cardiol 89(Pt B): 241-250.

19. Nakai A, Yamaguchi O, Takeda T, Higuchi Y, Hikoso S, et al. (2007) The role of autophagy in cardiomyocytes in the basal state and in response to hemodynamic stress. Nat Med 13(5): 619-624.

20. Hariharan N, Ikeda Y, Hong C, Alcendor R, Usui S, et al. (2013) Autophagy plays an essential role in mediating regression of hypertrophy during unloading of the heart. PLoS One 8(1): e51632.

21. Hill JA (2011) Autophagy in cardiac plasticity and disease. Pediatr Cardiol 32(3): 282-289.

22. Hariharan N, Maejima Y, Nakae J, Paik J, DePinho RD, et al. (2010) Deacetylation of FoxO by Sirt1 Plays an Essential Role in Mediating Starvation-Induced Autophagy in Cardiac Myocytes. Circ Res 107(12): 1470-1482.

23. Sandri M, Sandri C, Gilbert A, Skurk C, Calabria E, et al. (2004) Foxo transcription factors induce the atrophy-related ubiquitin ligase atrogin-1 and cause skeletal muscle atrophy. Cell 117(3): 399-412.

24. Porrello ER, Angelo D'Amore, Claire L Curl, Andrew M Allen, Stephen B Harrap, et al. (2009) Angiotensin II type 2 receptor antagonizes angiotensin II type 1 receptor-mediated cardiomyocyte autophagy. Hypertension 53(6): 1032-1040.

25. Weng, LQ Zhang Wb, Ye Y, Yin Pp, Yuan J, et al. (2014) Aliskiren ameliorates pressure overload-induced heart hypertrophy and fibrosis in mice. Acta Pharmacol Sin 35(8): 1005-1014.

26. Yue H, Li W, Desnoyer R, Sadashiva S Karnik (2010) Role of nuclear unphosphorylated STAT3 in angiotensin II type 1 receptor-induced cardiac hypertrophy. Cardiovasc Res 85(1): 90-99.

27. Zhao Z, Li Y, Wang M, Zhao S, Zhao Z, et al. (2020) Mechanotransduction pathways in the regulation of cartilage chondrocyte homoeostasis. J Cell Mol Med 24(10): 5408-5419.

28. Caramés B, Taniguchi N, Seino D, Blanco FJ, Darryl D’Lima, et al. (2012) Mechanical injury suppresses autophagy regulators and pharmacologic activation of autophagy results in chondroprotection. Arthritis Rheum 64(4): 1182-1192.

29. Nogueira-Recalde U, Lorenzo-Gomez I, Francisco J Blanco, María I Loza, Grassi D, et al. (2019) Fibrates as drugs with senolytic and autophagic activity for osteoarthritis therapy. E Bio Medicine 45: 588-605.

30.Xu HG, Yun-fei Yu, Quan Zheng, Wei Zhang, Chuang-dong Wang, et al. (2014) Autophagy protects end plate chondrocytes from intermittent cyclic mechanical tension induced calcification. Bone 66: 232-239.

31. Pan S (2009) Molecular mechanisms responsible for the atheroprotective effects of laminar shear stress. Antioxid Redox Signal 11(7): 1669-1682.

32. Guo F, Li X, Peng J, Tang Y, Yang Q, et al. (2014) Autophagy regulates vascular endothelial cell eNOS and ET-1 expression induced by laminar shear stress in an ex vivo perfused system. Ann Biomed Eng 42(9): 1978-1988.

33. Liu J, Bi X, Chen T, Zhang Q, Wang SX, et al. (2015) Shear stress regulates endothelial cell autophagy via redox regulation and Sirt1 expression. Cell Death Dis 6(7): e1827.

34. Ding Z, Liu S, Deng X, Fan Y, Wang X, et al. (2015) Hemodynamic shear stress modulates endothelial cell autophagy: Role of LOX-1. Int J Cardiol 184: 86-95.

35. Bharath LP, Mueller R, Li Y, Ruan T, Kunz D, et al. (2014) Impairment of autophagy in endothelial cells prevents shear-stress-induced increases in nitric oxide bioavailability. Can J Physiol Pharmacol 92(7): 605-612.

36. Gumucio JP, Davis MX, Bradley JR, Stafford PL, Schiffman CJ, et al. (2012) Rotator cuff tear reduces muscle fiber specific force production and induces macrophage accumulation and autophagy. J Orthop Res 30(12): 1963-1970. 
37. Ning L, Xu Z, Furuya N, Nonaka R, Yamada Y (2015) Perlecan inhibits autophagy to maintain muscle homeostasis in mouse soleus muscle. Matrix Biol 48: 26-35.

38. Ulbricht A, Felix J Eppler, Victor E Tapia, Peter F M van der Ven, Nico Hampe, et al. (2013) Cellular mechanotransduction relies on tensioninduced and chaperone-assisted autophagy. Curr Biol 23(5): 430-435.

39. Ulbricht A, Gehlert S, Leciejewski B, Schiffer T, Bloch W, et al. (2015) Induction and adaptation of chaperone-assisted selective autophagy

ISSN: 2574-1241

DOI: 10.26717/BJSTR.2020.32.005291

Yanghui Xing. Biomed J Sci \& Tech Res

(C) $(7)$ This work is licensed under Creative

Submission Link: https://biomedres.us/submit-manuscript.php
CASA in response to resistance exercise in human skeletal muscle. Autophagy 11(3): 538-546.

40. Chen Z, Fu Q, Shen B, Huang X, Wang K, et al. (2014) Enhanced p62 expression triggers concomitant autophagy and apoptosis in a rat chronic spinal cord compression model. Mol Med Rep 9(6): 2091-2096.

41. Park Y, Liu C, Luo T, Dietrich WD, Bramlett H, et al. (2015) ChaperoneMediated Autophagy after Traumatic Brain Injury. J Neurotrauma 32(19): 1449-1457.

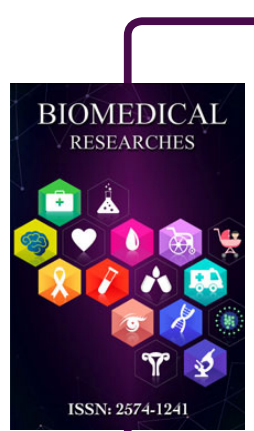

Assets of Publishing with us

- Global archiving of articles

- Immediate, unrestricted online access

- Rigorous Peer Review Process

- Authors Retain Copyrights

- Unique DOI for all articles

https://biomedres.us/ 